Zhang, Shuai, Yan, Yong, Qian, Xiangchen, Huang, Rong and Hu, Yonghui (2017) Homogenization of the Spatial Sensitivity of Electrostatic Sensors for the Flow Measurement of Pneumatically Conveyed Solids in a Square-Shaped Pipe. IEEE Sensors Journal, 17 (22). pp. 7516-7525. ISSN 1530-437X.

\title{
Downloaded from
}

https://kar.kent.ac.uk/63575/ The University of Kent's Academic Repository KAR

The version of record is available from

https://doi.org/10.1109/JSEN.2017.2758442

This document version

Author's Accepted Manuscript

DOI for this version

Licence for this version

UNSPECIFIED

\section{Additional information}

\section{Versions of research works}

\author{
Versions of Record \\ If this version is the version of record, it is the same as the published version available on the publisher's web site. \\ Cite as the published version.
}

\section{Author Accepted Manuscripts}

If this document is identified as the Author Accepted Manuscript it is the version after peer review but before type setting, copy editing or publisher branding. Cite as Surname, Initial. (Year) 'Title of article'. To be published in Title of Journal, Volume and issue numbers [peer-reviewed accepted version]. Available at: DOI or URL (Accessed: date).

\section{Enquiries}

If you have questions about this document contact ResearchSupport@kent.ac.uk. Please include the URL of the record in KAR. If you believe that your, or a third party's rights have been compromised through this document please see our Take Down policy (available from https://www.kent.ac.uk/guides/kar-the-kent-academic-repository\#policies). 


\title{
Homogenization of the Spatial Sensitivity of Electrostatic Sensors for the Flow Measurement of Pneumatically Conveyed Solids in a Square-Shaped Pipe
}

\author{
Shuai Zhang, Student Member, IEEE, Yong Yan, Fellow, IEEE, Xiangchen Qian, Member, IEEE, Rong \\ Huang, and Yonghui Hu, Member, IEEE
}

\begin{abstract}
The spatial sensitivity of an electrostatic sensor is recognized as an important factor that affects the accuracy of solids flow measurement in a pneumatic conveying pipe. However, the distribution of the spatial sensitivity is generally inhomogeneous due to the physical structure of the electrostatic sensor and the inherent electrostatic sensing mechanism. This paper proposes a sensitivity homogenization method based on differential measurement, i.e., using the differential outputs from two electrodes with different axial widths. The validity of the sensitivity homogenization method for a square-shaped electrostatic sensing head, which consists of strip-shaped electrode arrays with different widths, is validated through mathematical analysis. Furthermore, the response of the electrostatic sensing head incorporating the sensitivity homogenization method to roping flow regimes was evaluated on a gravity-fed solids flow test rig. Results from both modeling and experimental tests indicate that the homogeneity of the spatial sensitivity is improved significantly. The mean non-uniformity of the outputs from the sensing head is $11.7 \%$ as a result of the homogenization method.
\end{abstract}

Index Terms - electrostatic sensor, spatial sensitivity, sensitivity homogenization, square-shaped pipe, gas-solids two-phase flow.

\section{INTRODUCTION}

$\mathrm{O}$ WING to the advantages of structural simplicity, strong robustness, high stability and low cost, electrostatic sensors have been widely utilized for gas-solids two-phase flow measurement, especially when particles are conveyed in a dilute phase suspension [1-3]. Pulverized fuel (PF) flow in pneumatic conveying pipes at power stations and steel production plants is a typical example of dilute gas-solids two-phase flow. The dynamics of the PF flow in pneumatic conveying pipes is very complex and various flow patterns may occur, including roping flow regimes $[4,5]$. However, the spatial sensitivity distribution of electrostatic sensors is non-uniform because of the inherent

This work was supported in part by the National Natural Science Foundation of China under Grant 61603135. Mr. S. Zhang would also like to thank the IEEE Instrumentation and Measurement Society for supporting this work via the 2016 Graduate Fellowship Award.

S. Zhang, X. Qian, R. Huang, and Y. Hu are with the School of Control and Computer Engineering, North China Electric Power University, Beijing 102206, China.

Y. Yan is with the School of Engineering and Digital Arts, University of Kent, Canterbury, Kent CT2 7NT, UK, and also with the School of Control and Computer Engineering, North China Electric Power University, Beijing 102206, China (e-mail: y.yan@kent.ac.uk). limitation of electrostatic induction, i.e., the sensors are more sensitive to charged particles closer to the electrodes [6, 7]. As a result, for the same volume of passing particles, outputs of the sensors depend on the spatial distribution of particles, i.e., the flow regime. For this reason, the non-uniform spatial sensitivity of electrostatic sensors adversely affects the measurement accuracy of gas-solids two-phase flow. To mitigate the influence of flow regime on the flow measurement accuracy, the spatial sensitivity of electrostatic sensors should be homogenized.

In recent years many researchers have attempted to characterize the sensitivity of electrostatic sensors with different shaped electrodes through theoretical and experimental studies [8-15]. However, very little work has been undertaken to achieve sensitivity homogenization of the electrostatic sensors. Yan et al. [16] developed a mathematical model of a ring-shaped electrode and proved that the sensitivity of electrodes with a higher axial width to diameter ratio is more uniform [17]. Zhang et al. [6] analyzed the spatial sensitivity of a ring-shaped electrode in both time and frequency domains through finite element method, and presented a preliminary method to homogenize the spatial sensitivity in the frequency domain with weight compensation. However, the viability of the proposed homogenization method was not evaluated [18].

To homogenize the spatial sensitivity of electrostatic sensors is a challenge and significant research is therefore required. In this paper, spatial sensitivity homogenization of the electrostatic sensors to be used in a square-shaped pneumatic conveying pipe is reported. Square-shaped pneumatic conveying pipes are commonly used to transport particulate materials in building ductworks, circulating fluidized beds, and some thermal power plants. Earlier research has demonstrated that electrostatic sensors with distributed strip-shaped electrodes are capable of characterizing the local PF flow in a square-shaped pipe [19]. Due to the four sharp corners of a square-shaped pipe, the electric field distribution [15] and the PF flow regime [20] in the pipe are more complex than those in a circular pipe. This paper proposes a novel homogenization method through differential sensing arrangement. Theoretical analysis is carried out to validate the effectiveness of the proposed method. Experimental evaluations of the performance of a square-shaped electrostatic sensing head incorporating the homogenization method are also presented. 


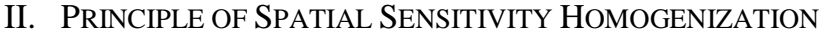

\section{A. Physical and Conceptual Models of the Sensing System}

As shown in Fig. 1(a), a square-shaped electrostatic sensing head composed of two distributed, strip-shaped electrode arrays (Array 1 and Array 2) with different widths in the flow direction was designed and implemented in this study. Each electrode array consists of 12 electrodes $(3 \times 4$, uniformly embedded in four flat pipe walls) with the same dimensions. Fig. 1(b) illustrates the principle of the differential sensing arrangement for spatial sensitivity homogenization. Outputs from the wide (Array 2) and narrow electrodes (Array 1) are amplified and filtered through two identical signal conditioning units. The signals from the narrow electrodes are multiplied by a weighing factor $k$ before being subtracted from the signals from the wide electrodes. The resulting signal is amplified further through a differential amplifier and then transmitted to a signal processing unit. The factor $k$ is associated with the characteristics of the original spatial sensitivity (OSS) of the strip electrodes and can be adjusted to generate the optimal differential spatial sensitivity (DSS) of the sensing head.

\section{Square-Shaped}

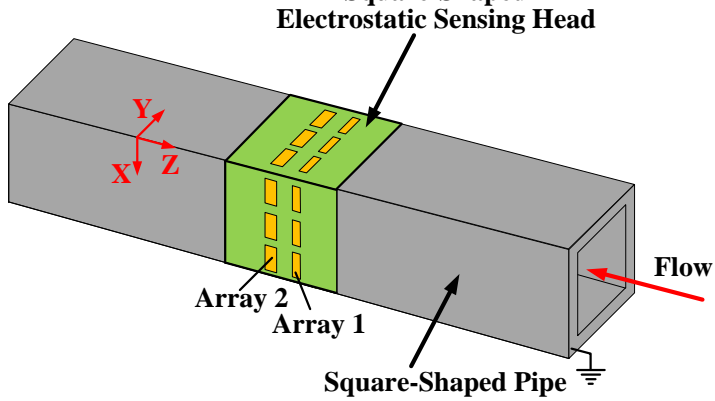

(a) Physical structure of the sensing head

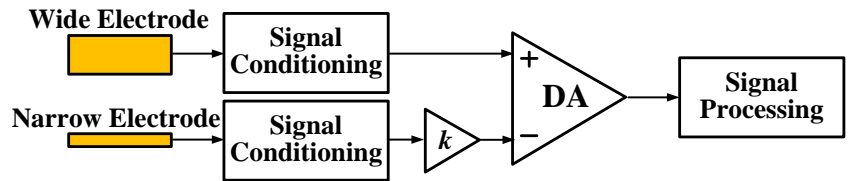

(b) Principle of differential sensing arrangement

Fig. 1. Physical structure of the sensing head and principle of the spatial sensitivity homogenization.

\section{B. Mathematical Model of the Sensing System}

A mathematical coordinate system for the sensing system using the method of images is shown in Fig. 2. The plane XOY lies on the median plane between the two electrode arrays, and the origin is located at one corner of the square-shaped pipe (Fig. 2(a)). The length of the electrodes on X-axis is marked as $l$, and the widths of the electrodes in Array 1 and Array 2 in the flow direction are $\mathrm{W} 1$ and $\mathrm{W} 2$, respectively. The center-to-center spacing between the two sets of electrode arrays in parallel is $L$. Suppose an ideal point charge with electric quantity $+q$ is placed in the square-shaped pipe. The induced charge and its fluctuations of representative electrodes $\mathrm{A}_{i}$ (adjacent to the corner area of the pipe cross section, $i=1,2$ ) and electrodes $\mathrm{B}_{i}$ (close to middle area, $i=1,2$ ) are derived in this study. The electric field in the square-shaped pipe is very complex due to the structure of the confined space. Based on the theories of electrostatics and the associated method of images [21], for the electrodes $\mathrm{B}_{i}$, the inner wall of the pipe (on XOY plane) can be regarded as an infinite grounded conducting plane (compared to the dimensions of the point charge and electrodes) with zero potential and the electric crosstalk between electrodes $\mathrm{B}_{i}$ and the other three pipe walls is insignificant and hence neglected. However, for electrodes $\mathrm{A}_{i}$, the electric crosstalk with the nearby pipe wall (on YOZ plane) is non-negligible, especially for the point charge located in the corner area close to the origin. The simplified image charges for electrodes $\mathrm{B}_{i}$ and $\mathrm{A}_{i}$ due to a point charge are depicted in Fig. 2(b) and Fig. 2(c), respectively.

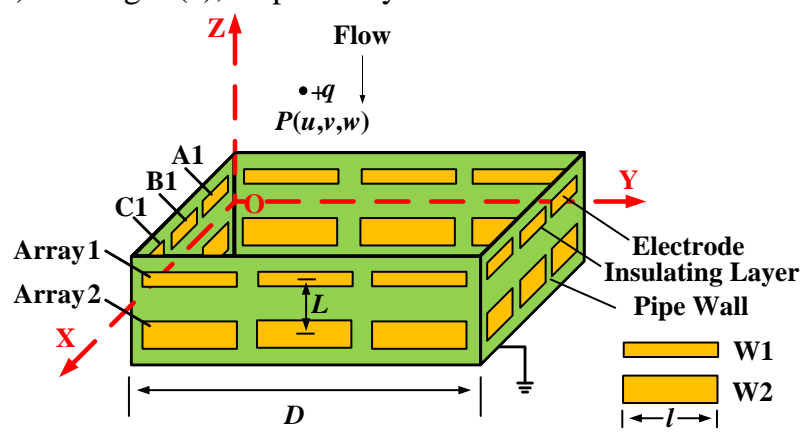

(a) Mathematical coordinate system

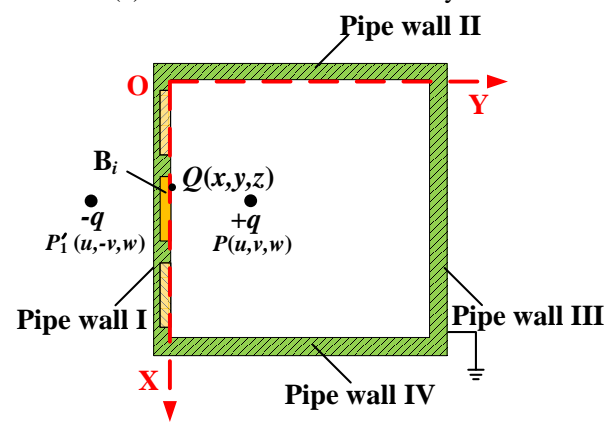

(b) Image charge for electrodes $\mathrm{B}_{i}$

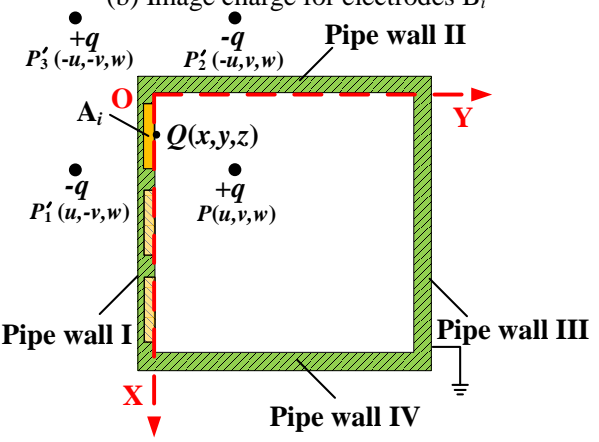

(c) Image charges for electrodes $\mathrm{A}_{i}$

Fig. 2. Mathematical coordinate system for the sensing system.

Suppose the source point charge with electric quantity $+q$ is located at $P(u, v, w)$ in the square-shaped pipe, and a given point $Q(x, y, z)$ is close to the surface of the electrodes. As for electrodes $\mathrm{B}_{i}$, the given point $Q$ is in the electric field due to the source point charge $P(u, v, w)$ and the image charge $P_{1}^{\prime}(u,-v, w)$ (with electric quantity $-q$ ), and for electrodes $\mathrm{A}_{i}$, another two image charges, $P_{2}^{\prime}(-u, v, w)$ with electric quantity $-q$ and $P_{3}^{\prime}(-u,-v, w)$ with electric quantity $+q$, also affect the electric field due to the existence of mutually perpendicular pipe walls (Pipe walls I and II). The electric potential at point $Q$ close to 
electrodes $\mathrm{B}_{i}$ and $\mathrm{A}_{i}$ (namely $\varphi_{B}$ and $\varphi_{A}$ respectively) is described as $[19,21]$ :

$$
\begin{gathered}
\varphi_{B}=\frac{q}{4 \pi \varepsilon|P Q|}+\frac{-q}{4 \pi \varepsilon\left|P_{1}^{\prime} Q\right|} \\
\varphi_{A}=\frac{q}{4 \pi \varepsilon|P Q|}+\frac{-q}{4 \pi \varepsilon\left|P_{1}^{\prime} Q\right|}+\frac{-q}{4 \pi \varepsilon\left|P_{2}^{\prime} Q\right|}+\frac{q}{4 \pi \varepsilon\left|P_{3}^{\prime} Q\right|}
\end{gathered}
$$

The surface charge density induced on electrodes $\mathrm{B}_{i}$ and $\mathrm{A}_{i}$ (marked as $\sigma_{B}$ and $\sigma_{A}$ respectively) on XOZ plane in response to the source point charge is given as follows:

$$
\begin{gathered}
\sigma_{B}=-\left.\varepsilon \frac{\partial \varphi_{B}}{\partial y}\right|_{y=0}=\frac{-q v}{2 \pi\left[(u-x)^{2}+v^{2}+(w-z)^{2}\right]^{3 / 2}} \\
\sigma_{A}=-\left.\varepsilon \frac{\partial \varphi_{A}}{\partial y}\right|_{y=0} \\
=\frac{q v}{2 \pi}\left\{\frac{1}{\left[(u+x)^{2}+v^{2}+(w-z)^{2}\right]^{3 / 2}}-\frac{1}{\left[(u-x)^{2}+v^{2}+(w-z)^{2}\right]^{3 / 2}}\right\}
\end{gathered}
$$

The induced charge $q^{\prime}(u, v, w)$ of electrodes $\mathrm{B}_{i}$ and $\mathrm{A}_{i}$ is thus determined from:

$$
q^{\prime}(u, v, w)=\int_{a}^{b} \int_{z_{1}}^{z_{2}} \sigma_{j} d x d z
$$

where $\sigma_{j}$ means $\sigma_{B}$ or $\sigma_{A}$ respectively, $a$ and $b$ are the coordinates of both ends of the electrode on X-axis $(b-a=l), z_{1}$ and $z_{2}$ are the coordinates of both ends of the electrode on $\mathrm{Z}$-axis and $z_{2}-z_{1}$ equals to the width of the electrode. The charge difference $q_{D}^{\prime}(u, v, w)$ between the induced charge $q_{2}^{\prime}$ on the electrodes in Array 2 and the induced charge $q_{1}^{\prime}$ on the electrodes in Array 1 with factor $k$ is determined accordingly from:

$$
q_{D}^{\prime}(u, v, w)=q_{2}^{\prime}-k q_{1}^{\prime}=\int_{a}^{b} \int_{\frac{-L-W 2}{2}}^{-L+W 2} \sigma_{j} d x d z-k \int_{a}^{b} \int_{\frac{L-W 1}{2}}^{L+W 1} \sigma_{j} d x d z \text { (6) }
$$

Since the spatial sensitivity of an electrode is defined as the absolute value of the ratio of induced charge $q^{\prime}$ and source charge $q[8,16]$, the OSS, $S(u, v, w)$, of each individual electrode, and the DSS, $S_{D}(u, v, w)$, of the sensing system are defined as, respectively:

$$
\begin{aligned}
& S(u, v, w)=\left|\frac{q^{\prime}(u, v, w)}{q}\right| \\
& S_{D}(u, v, w)=\left|\frac{q_{D}^{\prime}(u, v, w)}{q}\right|
\end{aligned}
$$

\section{Characteristics OF THE DifFERENTIAL SENSING SYSTEM}

\section{A. Distribution of the Differential Spatial Sensitivity}

A square-shaped electrostatic sensing head with an inner side length of the pipe wall $D=54 \mathrm{~mm}$ was implemented. The length of each electrode $(l)$ is $15 \mathrm{~mm}$ and the widths of the electrodes in the two arrays are $6 \mathrm{~mm}$ (W1, in Array 1) and $9 \mathrm{~mm}$ (W2, in Array 2), respectively. To ensure a pair of differential electrodes in two arrays senses the same charged particle synchronously, the median planes of the two electrode arrays should ideally coincide, i.e., the center-to-center spacing ( $L$, Fig. 2(a)) equals zero. However, due to the limitations of the physical construction of the sensing head, $L$ should be greater than (W1+W2)/2. The center-to-center spacing is set as $15 \mathrm{~mm}$ in this study. The dimensions of the electrodes in one side of the sensing head (electrodes A1 to C2) are illustrated in Fig. 3.

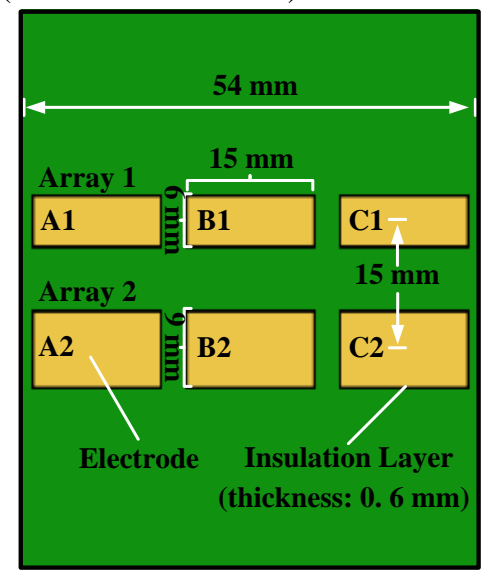

Fig. 3. Dimensions of the electrodes in one side of the sensing head.

Since the electrostatic sensors are more sensitive to the charge on the median plane of the electrode $[8,15]$, three specific planes are chosen to characterize the spatial sensitivity distribution, i.e., median plane of electrode Array $1(z=L / 2$, namely S1), median plane of electrode Array 2 ( $z=-L / 2$, marked as S2), and median plane of the sensing head (XOY plane, $z=0$, marked as S0). To quantify the sensitivity homogeneity of the sensing system, a parameter called non-uniformity, $H_{r}$, is introduced here:

$$
H_{r}=\Delta S_{\max }
$$

where $\Delta S_{\max }$ is the maximum sensitivity deviation of an electrode over the square cross section. The smaller $H_{r}$, the more uniform the spatial sensitivity. $H_{r}$ of the DSS for electrodes $\mathrm{B}_{i}$ and $\mathrm{A}_{i}$ on $\mathrm{S} 0$ plane with $k$ in range of 0-3 is shown in Fig. 4.

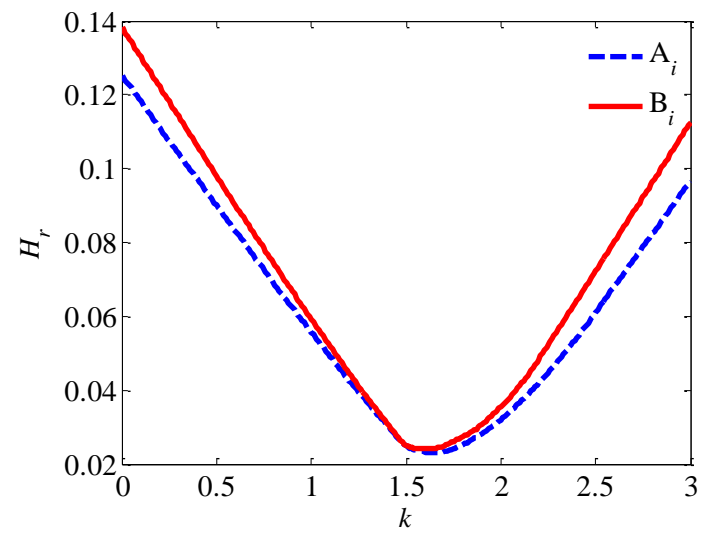

Fig. 4. Non-uniformity of DSS on XOY plane.

It can be observed that $H_{r}$ decreases with factor $k$ when $k$ $<1.6$ and then increases when $k>1.6$. However, if the factor $k$ is greater than 1, negative differential outputs from the sensing system may arise in the tests when particles are conveyed with a 
low mass flow rate. This is due to the fact that the outputs from the wide and narrow electrodes for particles far from the electrodes are very close, i.e., the data presented in Fig. 10(b) (part B of Section IV). For this reason, $k$ is set to 1 in this study.

The OSS of electrodes B1 and A1 on plane S1 (marked as $S_{B I}(u, v, w)$ and $\left.S_{A I}(u, v, w)\right)$, OSS of electrodes B2 and A2 on plane S2 (marked as $S_{B 2}(u, v, w)$ and $S_{A 2}(u, v, w)$ ), and the DSS between electrodes $\mathrm{B}_{i}$ and $\mathrm{A}_{i}$ on plane $\mathrm{S} 0$ (marked as $S_{D B}(u, v, w)$ and $\left.S_{D A}(u, v, w)\right)$ are illustrated in Fig.5.

It is clear that the OSS distribution of strip-shaped electrodes is highly non-uniform, i.e., the spatial sensitivity near the

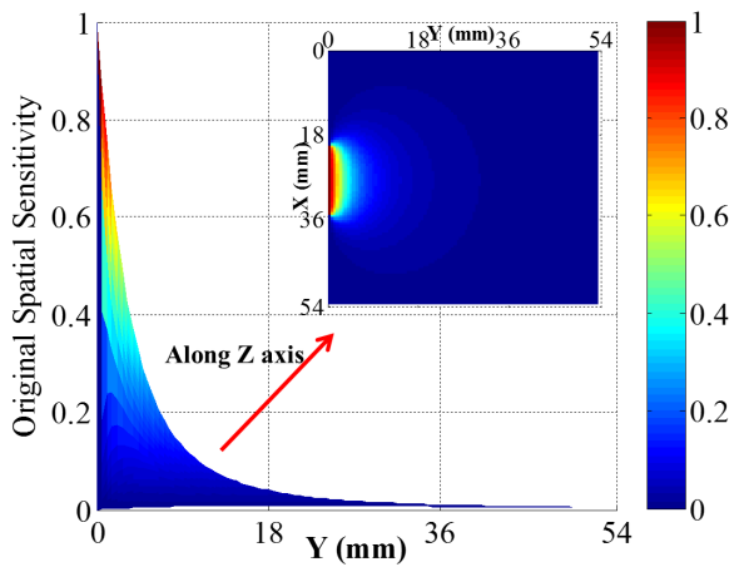

(a) $S_{B I}(u, v, w)$

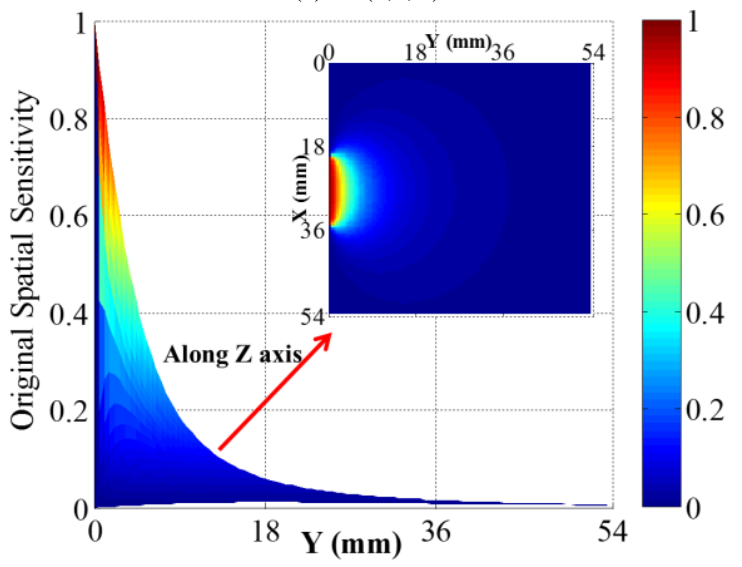

(c) $S_{B 2}(u, v, w)$

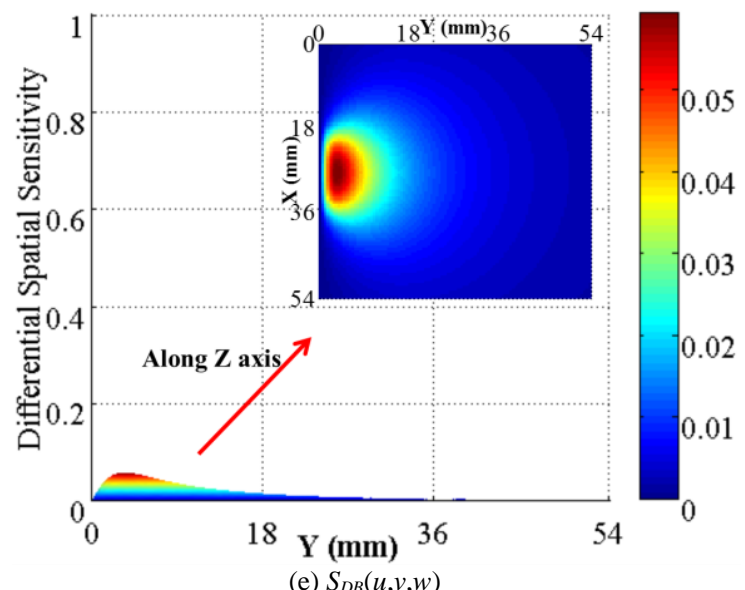

surface of the electrode is close to 1 and decreases sharply with the distance away from the electrode. This reduction tendency for the wider electrodes (electrodes B2 and A2) is relatively slower. Meanwhile, although the strip-shaped electrode is arranged along the $\mathrm{X}$-axis, its sensitivity distribution is not parallel to the $\mathrm{X}$ axis because of the fringe effect of the electrode, especially for the electrode in the corner (electrodes $\mathrm{A}_{i}$ ). Compared to these trends, the DSS (Fig. 5(e) and Fig. 5(f)) is more homogenous. To emphasize the surface slopes of the OSS and DSS in Fig. 5, their gradients are calculated and depicted in Fig. 6.

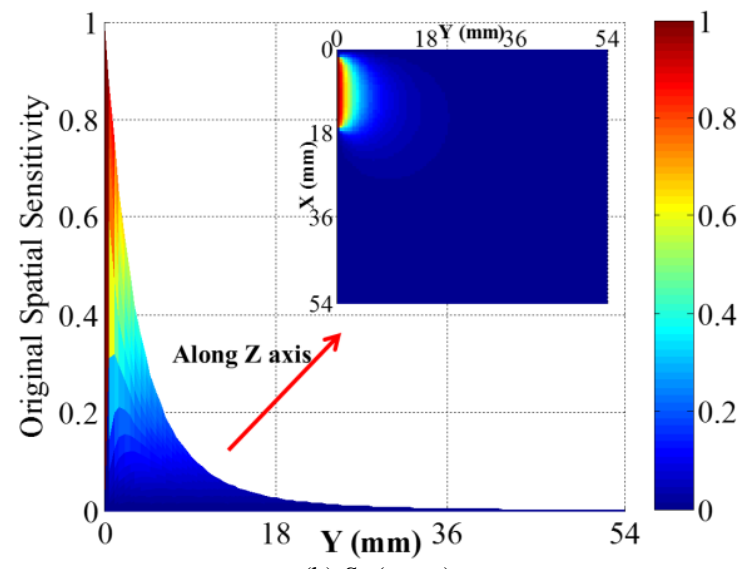

(b) $S_{A l}(u, v, w)$

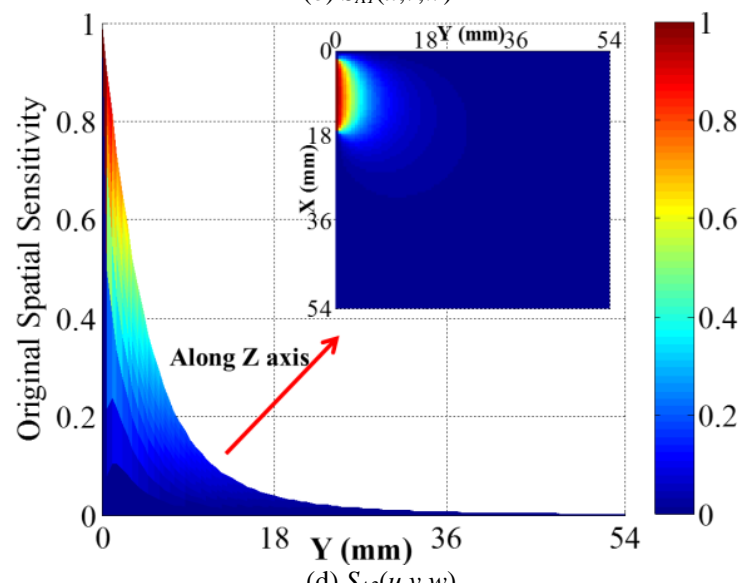

(d) $S_{A 2}(u, v, w)$

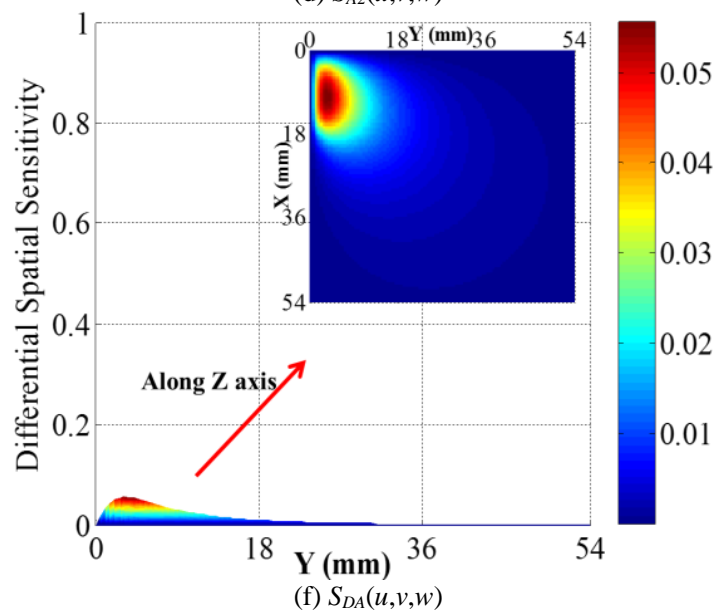

Fig. 5. Distribution of OSS and DSS 


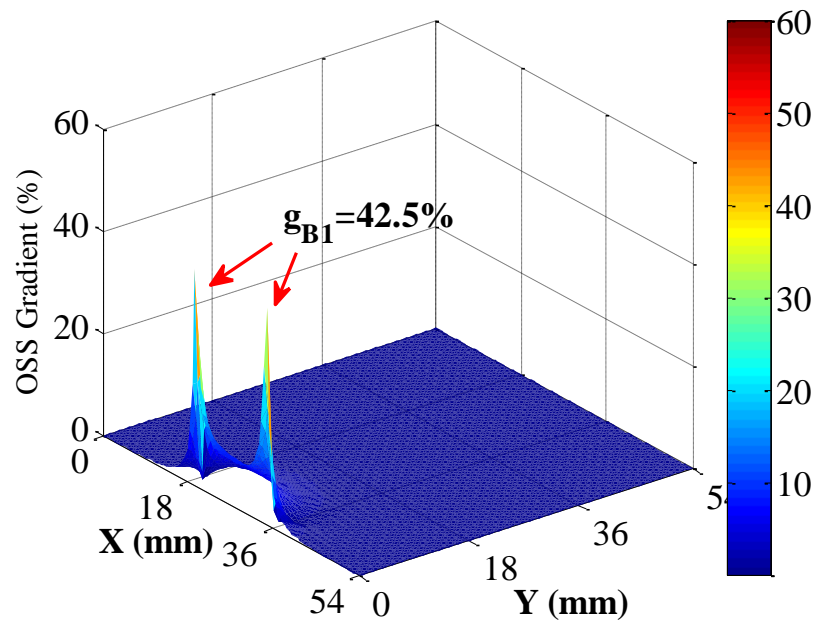

(a) $S_{B I}(u, v, w)$

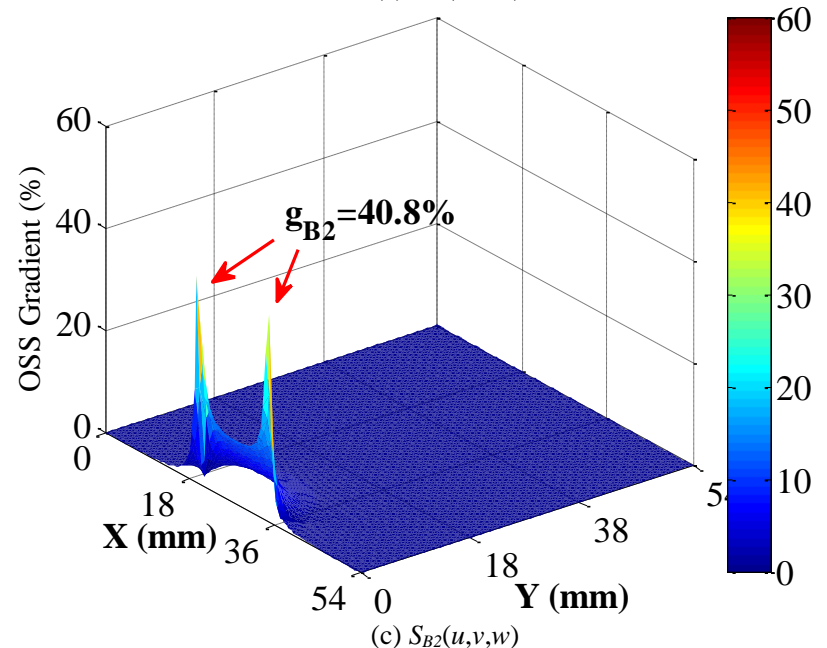

(c) $S_{B 2}(u, v, w)$

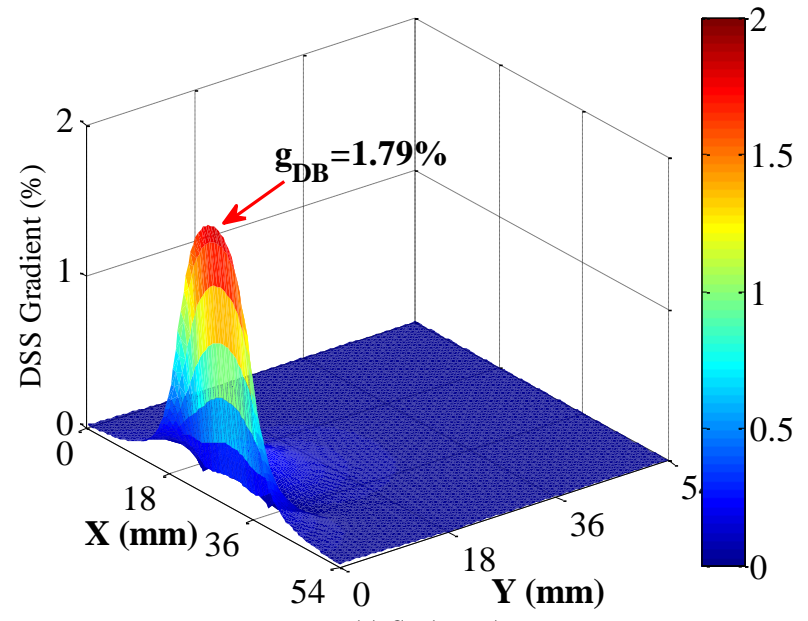

(e) $S_{D B}(u, v, w)$

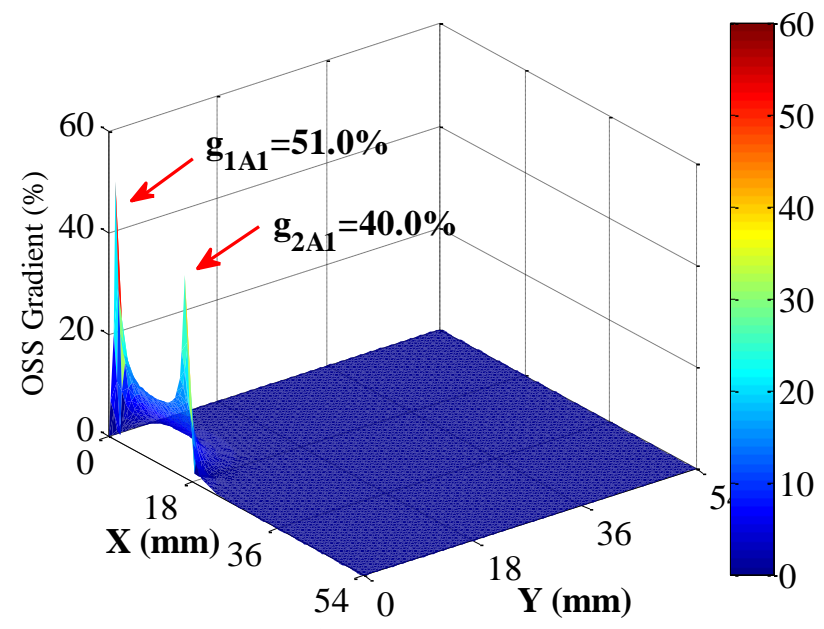

(b) $S_{A I}(u, v, w)$
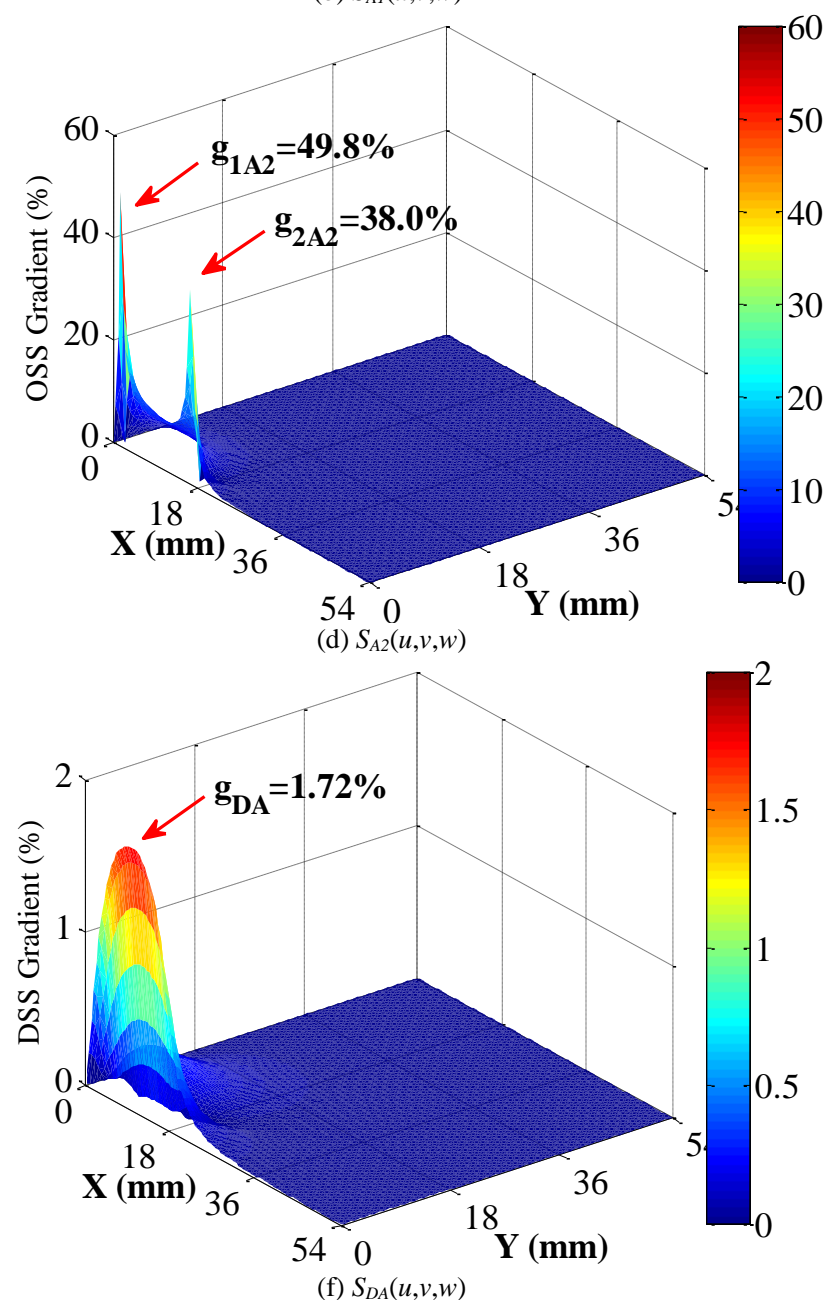

Fig. 6. Gradients of OSS and DSS.

Fig. 6(a)-(d) show that the slopes of the OSS for electrodes $\mathrm{B}_{i}$ and $\mathrm{A}_{i}$ in the close vicinity of the electrodes are steep, but relatively smooth in other areas. Meanwhile, two sharp peaks exist near both ends of the electrodes and wider electrodes yield less peaks. For electrodes $\mathrm{B}_{i}(i=1,2)$, the two gradient peaks are equal due to the symmetrical arrangement of the electrodes. For electrodes $\mathrm{A}_{i}$, however, the gradient peak closer to the corner is higher than the other end. These trends demonstrate that the OSS in the corners is less uniform. The gradients of the DSS, as shown in Fig. 6(e) and Fig. 6(f), are much less than either of the OSS over the whole cross-section of the square pipe. Consequently, the DSS of the sensing system is significantly more uniform than the OSS of the individual electrodes, though both the OSS and DSS have a higher magnitude in areas closer the electrodes. 


\section{B. Non-uniformity of Differential Spatial Sensitivity}

Suppose the cross-section of the square pipe is divided into nine measurement zones (Fig. 7), i.e., four corner zones (Zones I, III, VII and IX), four zones along the pipe walls (Zones II, IV, VI and VIII), and one central zone (Zone V). To compare the non-uniformity of the OSS and DSS over the nine measurement zones, the mean OSS in each zone for electrodes B1 and A1 on plane $\mathrm{S} 1$ and for electrodes $\mathrm{B} 2$ and $\mathrm{A} 2$ on plane $\mathrm{S} 2$ and mean DSS on plane S0 are shown in Fig. 8. Each data point presented in Fig.8 is the average of the modeling data in the corresponding zone.
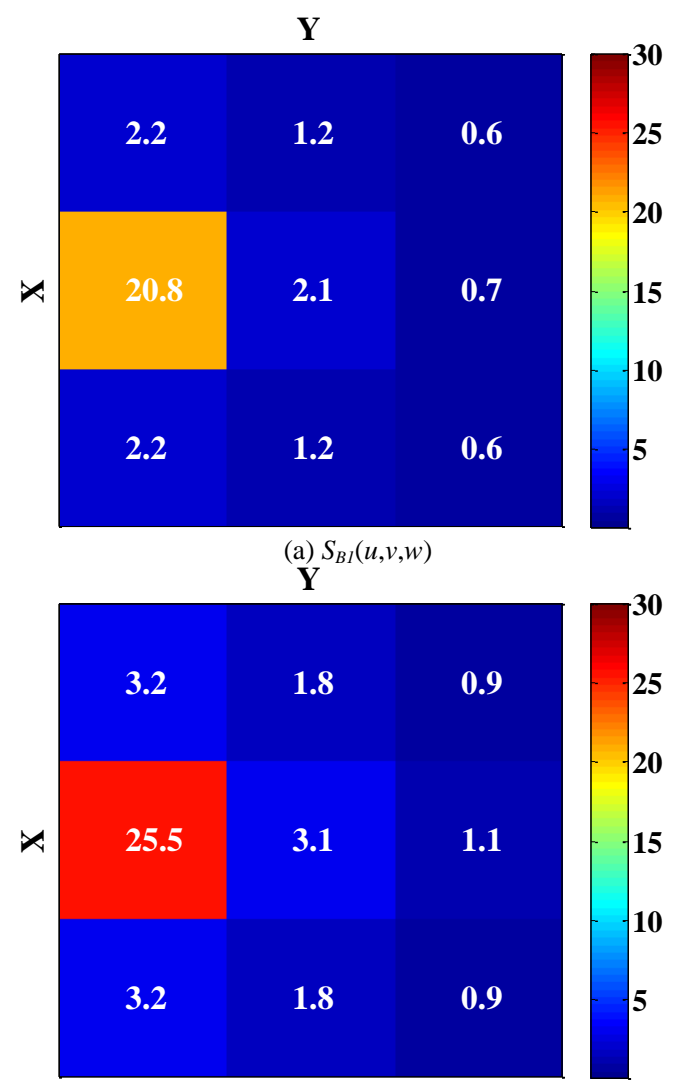

(c) $S_{B 2}(u, v, w)$

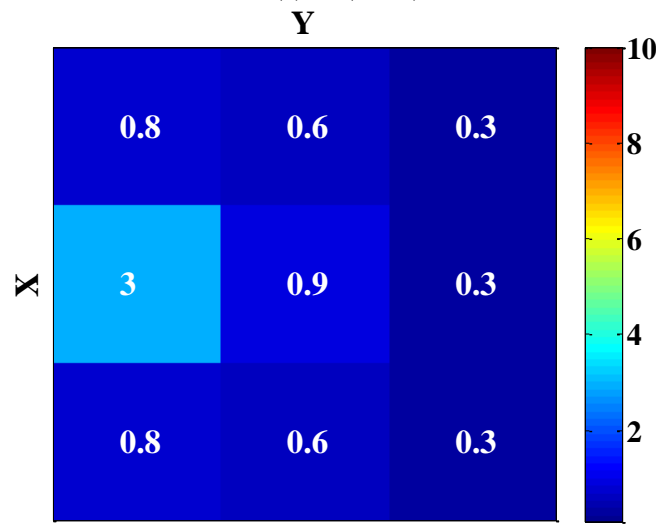

(e) $S_{D B}(u, v, w)$

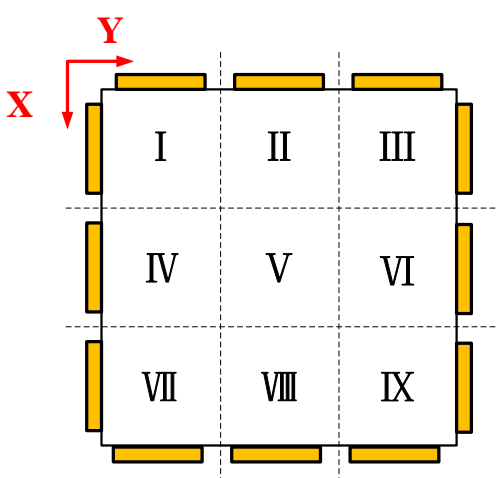

Fig. 7. Measurement zone partitions over the pipe cross-section.

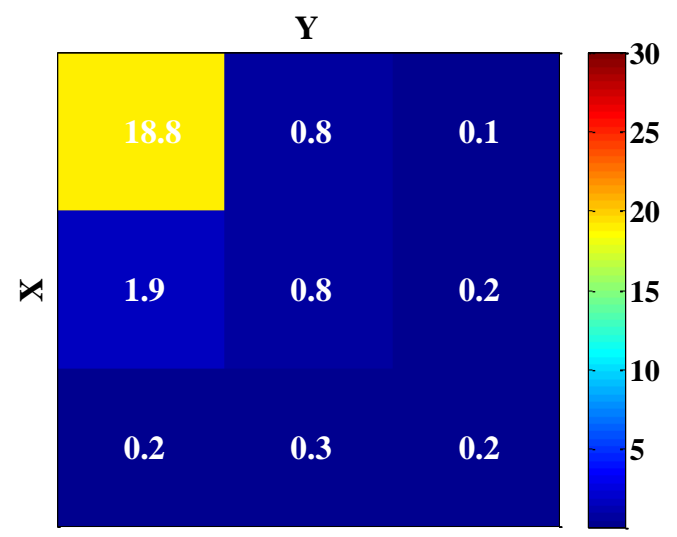

(b) $S_{A I}(u, v, w)$

$\mathbf{Y}$

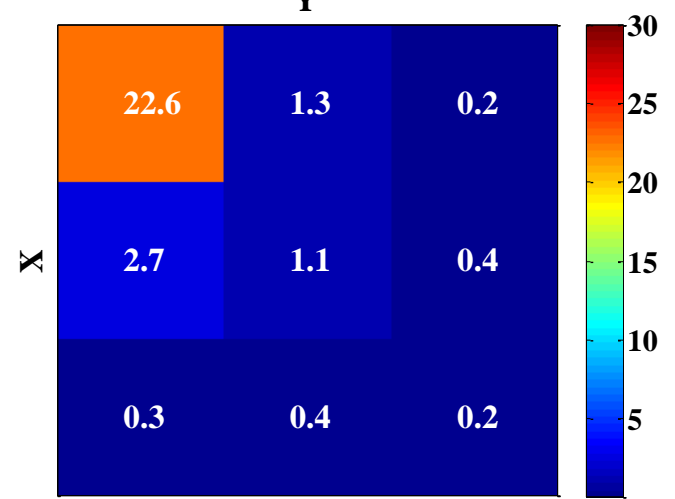

(d) $S_{A 2}(u, v, w)$

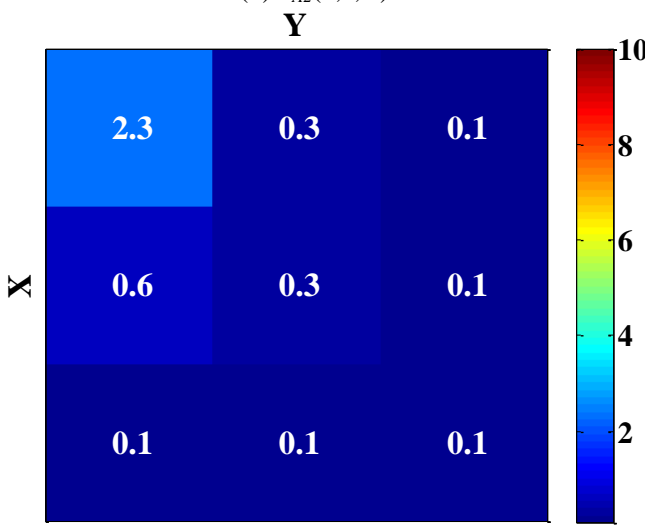

(f) $S_{D A}(u, v, w)$

Fig. 8. Mean OSS and DSS of modeling data (\%). 
It is evident from Fig. 8 that both the OSS and DSS of the electrodes in the zones nearest to the electrodes are the highest. The DSS is weaker in amplitude over the whole cross-section, but is more homogenous than the OSS. In practice, the drawback of the non-intrusive electrostatic sensors is the highly non-uniform spatial sensitivity across the whole sensing zone. The weaker overall sensitivity of the DSS can be compensated through the provision of a high-quality pre-amplifier with a suitably higher gain in the signal conditioning unit in order to maintain an adequate level of signals prior to the signal processing unit (Fig. 1(b)). The $H_{r}$ of the mean OSS and DSS in Fig. 8 is summarized in Table I (normalized to the maximum spatial sensitivity of electrodes B2 and A2, respectively). As can be seen from Table I, the maximum $H_{r}$ of the DSS is $2.7 \%$, which is less than $1 / 7$ of the OSS. The non-uniformity of the DSS for the sensing system is significantly reduced from that of the OSS.

TABLE I

NON-UNIFORMITY OF SPATIAL SENSITIVITY (MODELING DATA)

\begin{tabular}{ccc}
\hline \multirow{2}{*}{ Sensitivity } & \multicolumn{2}{c}{$H_{r}(\%)$} \\
& Electrodes $\mathrm{B}_{i}$ & Electrodes $_{i}$ \\
\hline$S_{B I / A 1}$ & 20.3 & 18.7 \\
$S_{B 2 / A 2}$ & 24.6 & 22.4 \\
$S_{D B / D A}$ & 2.7 & 2.2 \\
\hline
\end{tabular}

\section{EXPERIMENTAL RESULTS AND DISCUSSION}

\section{A. $\quad$ Test Rig}

A purpose-built gravity-fed test rig with the square-shaped electrostatic sensing head is shown in Fig. 9. The dimension of the inner side of the square-shaped pipe section is $54 \mathrm{~mm}$. The guide tube is made of stainless steel and has a length of 1500 $\mathrm{mm}$ with an inner diameter of $14 \mathrm{~mm}$. Fine silica sand with a mean diameter of $90 \mu \mathrm{m}$ (measured using a laser particle size analyzer, Model OMEC LOP9) was used as test particles. The sand particles were dropped from a hopper on the top of the guide tube and were confined within the guide tube to form a well-defined roping flow regime. Since the roping flow regime is an extreme pattern of inhomogeneous solids distribution, in which particles are highly concentrated in a small area of the cross section [4-6], it was used to characterize the sensitivity distribution of the sensing system in this study. The mass flow rate of particles was controlled by changing the hopper with a different sized outlet. The traversing path of the flow was controlled by adjusting the relative location of the guide tube from the sensing head. To avoid the effect of contact electrification [22], the electrodes were covered in a thin mask layer (liquid photoimageable solder mask).

\section{B. Experimental Results}

Three hoppers with different sized outlets were used to form three mass flow rates of particles at $17.2 \mathrm{~kg} / \mathrm{h}, 47.1 \mathrm{~kg} / \mathrm{h}$, and $83.1 \mathrm{~kg} / \mathrm{h}$ (marked as M1, M2, and M3), respectively. The ambient temperature was $19 \square \mathrm{C}$ and relative humidity of air was $17 \%$. The roping flow from the hopper was traversed along the central axes of the nine zones, respectively, by adjusting the holder. The experimental work was repeated for three times under each test condition. The signals were sampled at a rate of $10 \mathrm{kHz}$. The velocity of the particles passing through the sensing head was around $2.6 \mathrm{~m} / \mathrm{s}$, which was measured using an electrostatic velocimeter $[3,16]$. Since the root mean square (RMS) amplitude of a sensor signal reflects the strength of the output from the electrode [1,3], the RMS values of the outputs from the electrodes in Array 1 and differential outputs from electrodes A2 and A1 and electrodes B2 and B1, respectively, are depicted in Fig. 10. Each value in Fig.10 was averaged over 30 seconds under steady flow conditions.

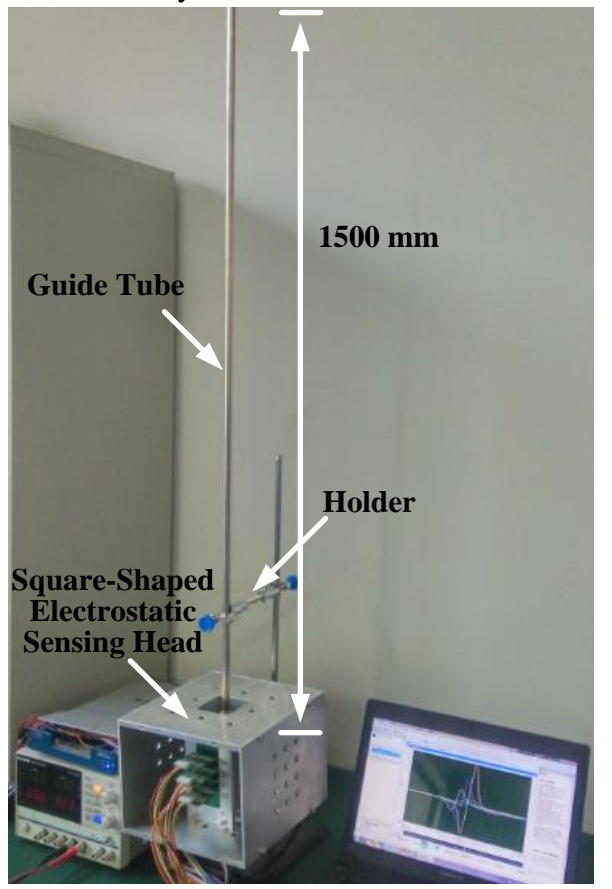

Fig. 9. Experimental set-up with the electrostatic sensing head.

Fig. 10 shows that both the RMS values of the outputs and differential outputs in the nearest zone (adjacent to corresponding electrodes) are the highest for the three mass flow rates of particles. This outcome agrees well with the modeling data presented in Fig. 8. Meanwhile, the outputs from the original electrodes and from the differential arrangement all increase with the mass flow rates of particles. The reduction in values along the $\mathrm{X}$-axis is smaller than that along the $\mathrm{Y}$-axis, and the RMS distributions are not as symmetrical as the modeling data due to minor uncontrollable factors during experimentation, such as asymmetrical traversing paths and pre-charge status of the particles during particle falling through the guide tube. The $H_{r}$ of the experimental data is summarized in Table II (normalized to the maximum outputs (RMS) from electrodes A2 and B2 under roping flow regimes in Zones I and IV accordingly). 


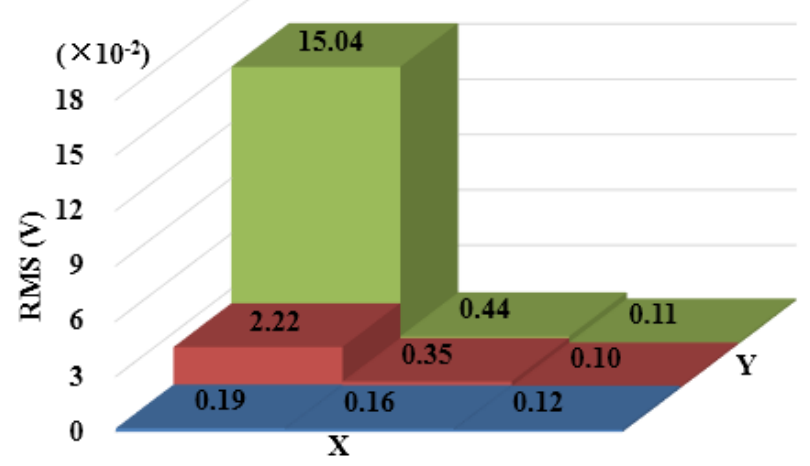

(a) Outputs from A1 for M1

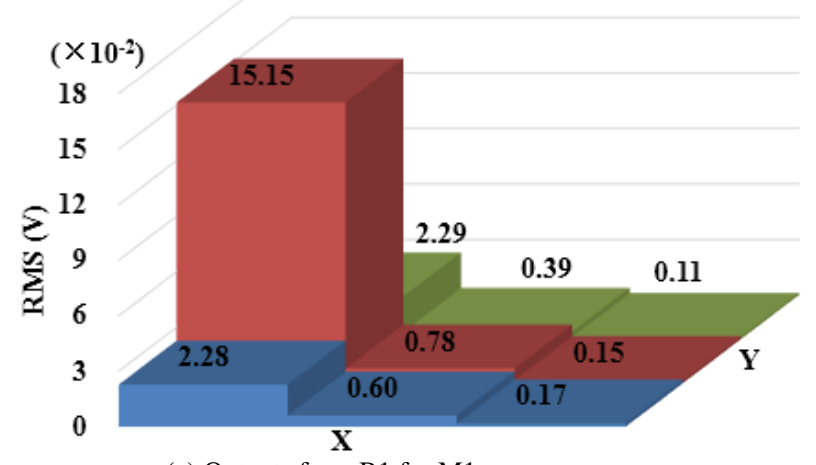

(c) Outputs from B1 for M1

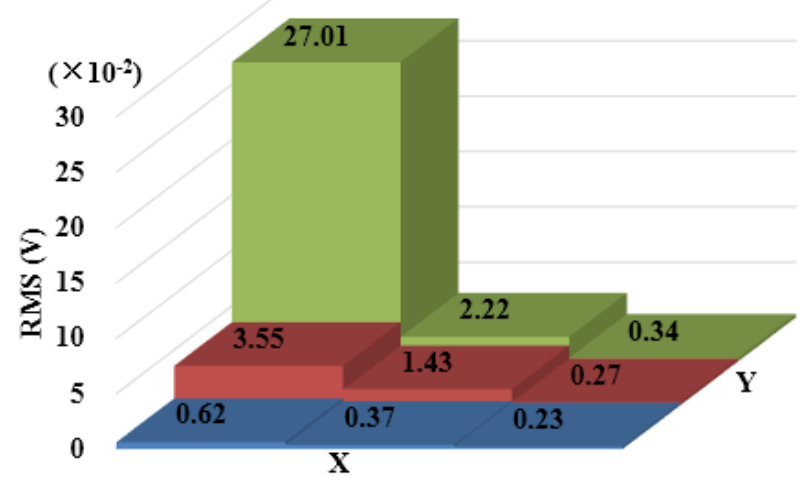

(e) Outputs from A1 for M2

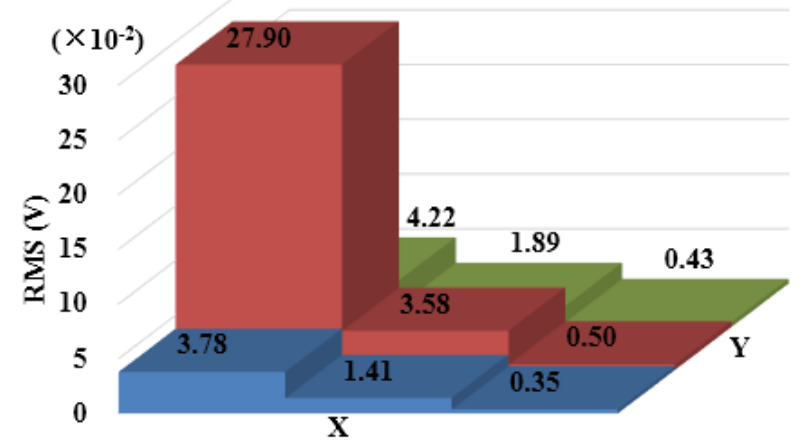

(g) Outputs from B1 for M2

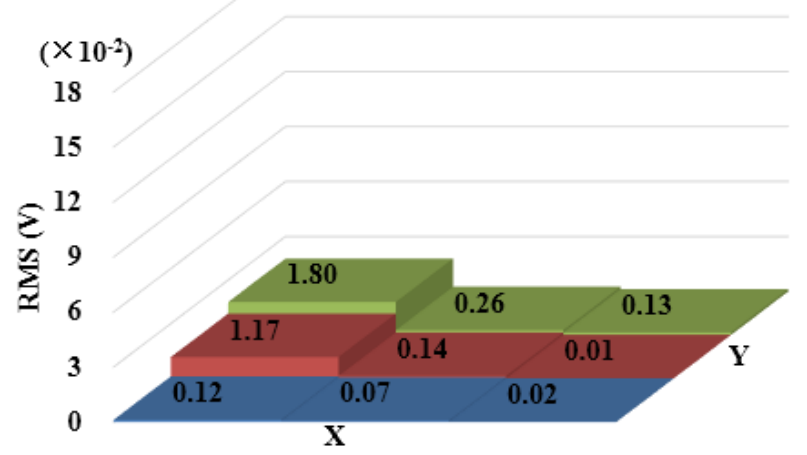

(b) Differential outputs from A2 and A1 for M1

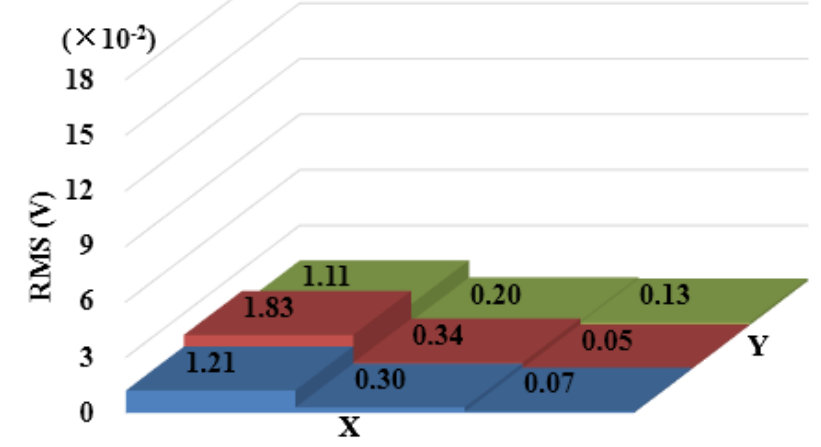

(d) Differential outputs from B2 and B1 for M1

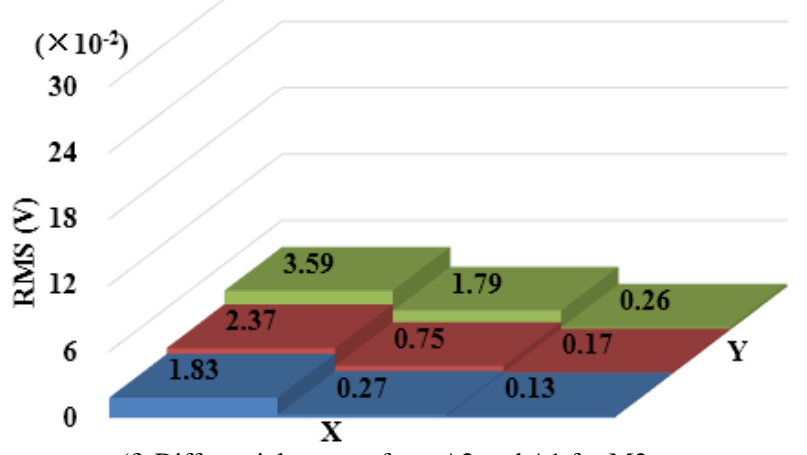

(f) Differential outputs from A2 and A1 for M2

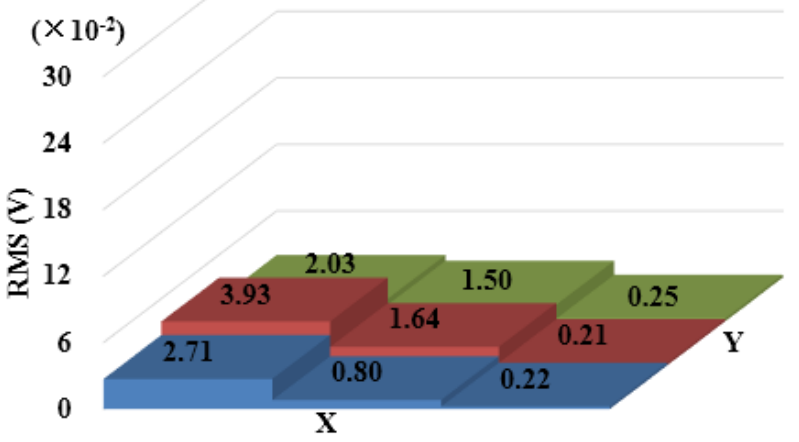

(h) Differential outputs from B2 and B1 for M2 


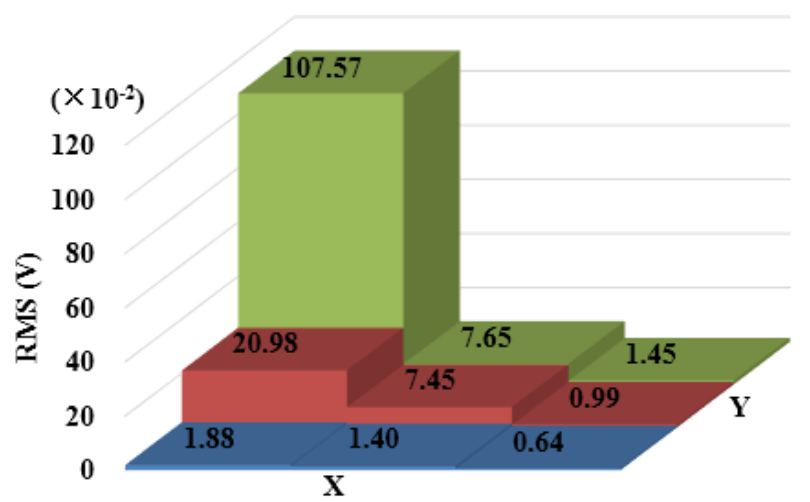

(i) Outputs from A1 for M3

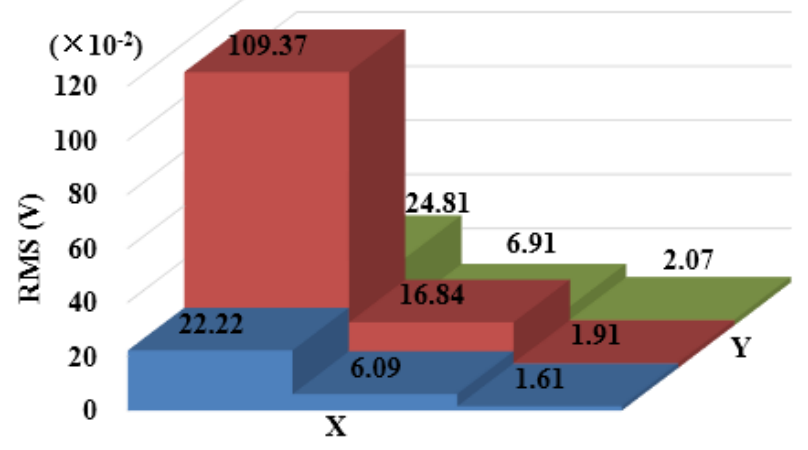

(k) Outputs from B1 for M3

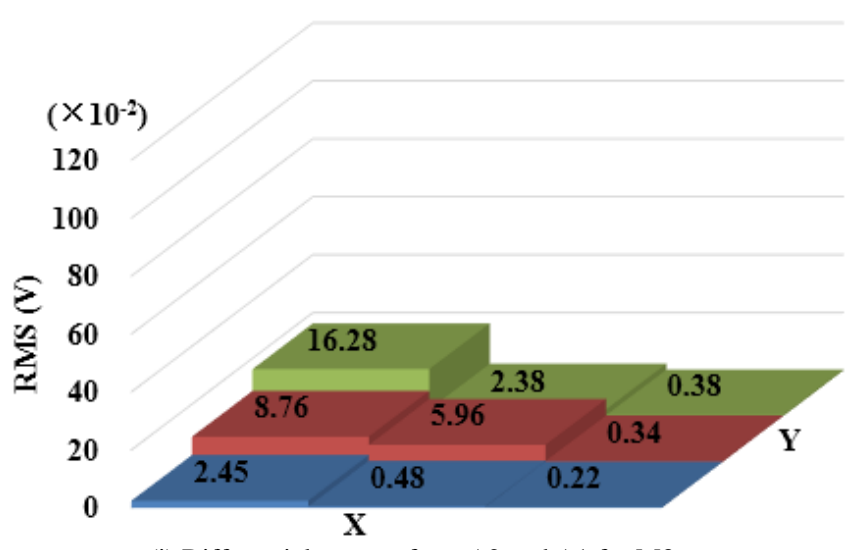

(j) Differential outputs from A2 and A1 for M3

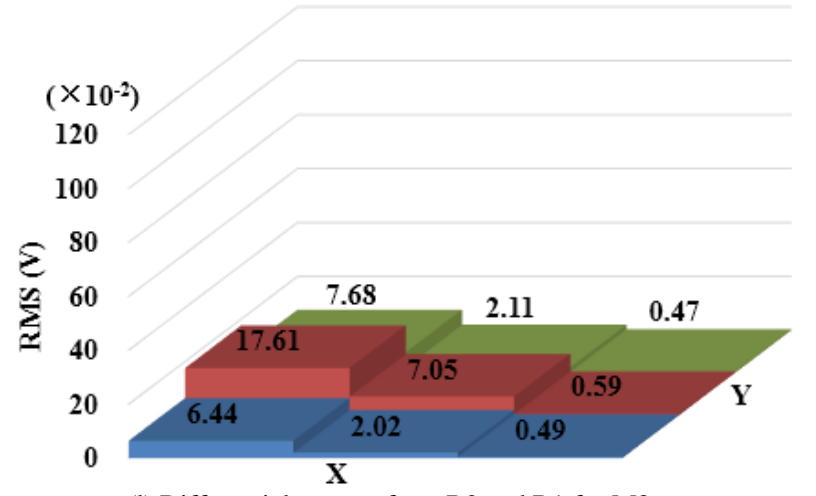

(1) Differential outputs from B2 and B1 for M3

Fig. 10. RMS of the outputs from electrodes in Array 1 and differential outputs from the sensing head for three different mass flow rates.

As can be seen from Table II, the non-uniformity of the sensing head in response to the roping flow regime is in the range of $10.5 \%-13.5 \%$ (mean $H_{r}=11.7 \%$ ), which is also less than $1 / 7$ of the original outputs $(84.9 \%-88.5 \%)$. The homogeneity of the sensing system is significantly improved.

TABLE II

NON-UNIFORMITY OF SENSING HEAD (EXPERIMENTAL DATA)

\begin{tabular}{cccc}
\hline \hline \multicolumn{2}{c}{ Signal } & \multicolumn{2}{c}{$H_{r}(\%)$} \\
& & Electrodes $\mathrm{B}_{i}$ & Electrodes $\mathrm{A}_{i}$ \\
\hline \multirow{2}{*}{$\mathrm{M} 1$} & $S_{B 1 / A l}$ & 88.7 & 88.5 \\
& $S_{D B / D A}$ & 10.6 & 10.5 \\
$\mathrm{M} 2$ & $S_{B 1 / A l}$ & 87.5 & 86.5 \\
& $S_{D B / D A}$ & 11.3 & 11.7 \\
\multirow{2}{*}{$\mathrm{M} 3$} & $S_{B 1 / A l}$ & 86.3 & 84.9 \\
& $S_{D B / D A}$ & 13.0 & 13.5 \\
\hline \hline
\end{tabular}

As for the frequency response of the sensing system, the amplitude spectra of the original outputs from electrodes A1, A2, B1, B2, independently, and the differential outputs from electrodes A2 and A1 (marked as DA) and electrodes B2 and B1 (marked as DB), for the roping flow with M2 mass flow rate at Zone I, are depicted in Fig. 11, respectively. These spectra are representative of results from many repeated experiments. Fig. 11 shows that electrodes B1 and B2 produce weaker signals than those from electrodes $\mathrm{A} 1$ and $\mathrm{A} 2$ as the flow is located in Zone I (next to electrodes A1 and A2), and that the wider electrodes produce stronger signals, which agree with earlier studies $[11,16]$. However, the amplitude spectra of the differential outputs, DA and DB, are not a direct linear differential combination of the amplitude spectra of the signals from electrodes $\mathrm{A}_{i}$ and $\mathrm{B}_{i}(i=1,2)$ due to the fact that the signals from each pair of arrays are asynchronous and hence have different phase spectra.

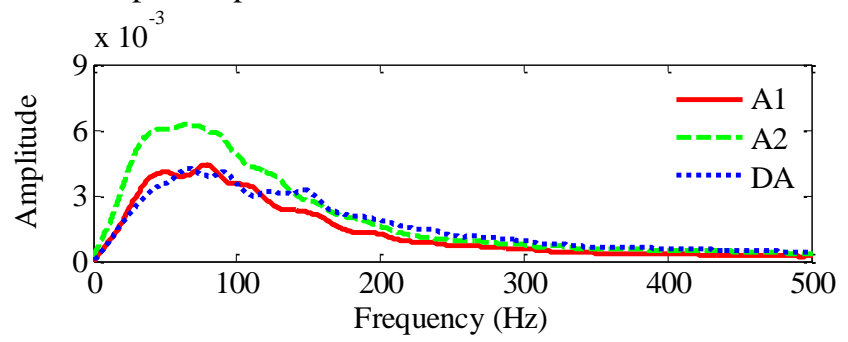

(a) Electrodes A1 and A2

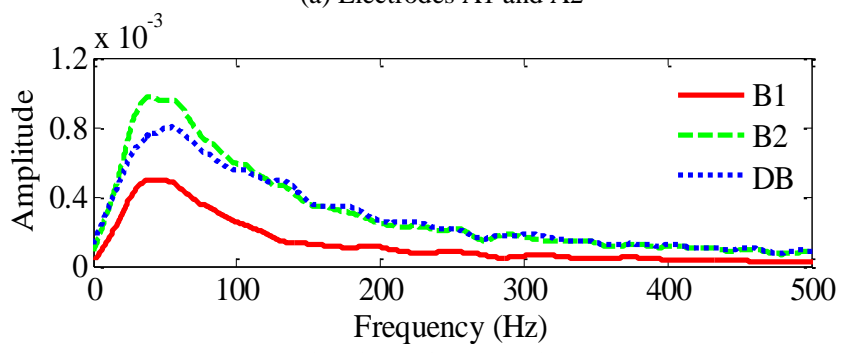

(b) Electrodes B1 and B2

Fig. 11. Amplitude spectra of the outputs from single electrodes and differential outputs from the sensing head.

\section{CONCLUSIOnS}

Based on both theoretical and experimental studies of a square-shaped electrostatic sensing head, a sensitivity 
homogenization method through differential sensing arrangement between two strip-shaped electrostatic electrodes with different widths in the flow direction is proposed in this paper. Modeling and experimental results presented have demonstrated that the homogeneity of the sensing head incorporating the method is improved significantly, despite that the sensing system is still more sensitive to particles closer to the electrodes. The mean non-uniformity of the sensing head obtained in the experiments with roping flow regimes is $11.7 \%$. This means inhomogeneous distribution of particles across the pipe section will have a little impact on the flow measurement of pneumatically conveyed solids, if the proposed homogenization method is effectively adopted in the sensing elements of the measurement system. It is envisaged that the homogenization method will provide useful guidance to the optimized design of electrostatic sensors for the flow measurement of pneumatically conveyed solids in square shaped or circular pipes.

\section{REFERENCES}

[1] J. B. Gajewski, "Electrostatic nonintrusive method for measuring the electric charge, mass flow rate, and velocity of particulates in the two-phase gas-solid pipe flows-its only or as many as 50 years of historical evolution," IEEE Trans. Ind. Appl., vol. 44, no. 5, pp. 1418-1430, 2008.

[2] Y. Zheng, and Q. Liu, "Review of techniques for the mass flow rate measurement of pneumatically conveyed solids," Measurement, vol. 44, no. 4, pp. 589-604, 2011.

[3] X. Qian, Y. Yan, X. Huang, and Y. Hu, "Measurement of the mass flow and velocity distributions of pulverized fuel in primary air pipes using electrostatic sensing techniques," IEEE Trans. Instrum. Meas. vol. 66, no. 5, pp. 944-952, 2017.

[4] D. Mills, "Pneumatic conveying design guide," Butterworth-Heinemann Pub., 2nd ed., Oxford, U.K., pp. 236-257, 2004.

[5] H. Hu, J. Dong, J. Zhang, Y. Cheng, and T. Xu, "Identification of gas/solid two-phase flow regimes using electrostatic sensors and neural-network techniques," Flow Meas. Instrum., vol. 22, no. 5, pp. 482-487, 2011.

[6] J. Zhang, and J. Coulthard, "Theoretical and experimental studies of the spatial sensitivity of an electrostatic pulverised fuel meter," J. Electrostat., vol. 63, no. 12, pp. 1133-1149, 2005.

[7] S. E. Law, "Electrostatic induction instrumentation for tracking and charge measurement of airborne agricultural particulates," ASAE Trans., vol. 18, no. 1, p. 40-45, 1975.

[8] C. Xu, S. Wang, G. Tang, D. Yang, and B. Zhou, "Sensing characteristics of electrostatic inductive sensor for flow parameters measurement of pneumatically conveyed particles" J. Electrostat., vol. 65, no. 9, pp. 582-592, 2007.

[9] S. N. Murnane, R. N. Barnes, S. R. Woodhead, and J. E. Amadi-Echendu, "Electrostatic modelling and measurement of airborne particle concentration," IEEE Trans. Instrum. Meas., vol. 45, no. 2, pp. 488-492, 1996.

[10] M. F. Rahmat, and N. S. Kamaruddin, "An electrodynamic sensor for electrostatic charge measurement," Int. J. Smart Sensing Intelligent Syst., vol. 2, no. 2, pp. 200-212, 2009.

[11] C. Xu, J. Li, H. Gao, and S. Wang, "Investigations into sensing characteristics of electrostatic sensor arrays through computational modelling and practical experimentation," J. Electrostat., vol. 70, no. 1, pp. 60-71, 2012.

[12] J. Li, C. Xu, and S. Wang, "Local particle mean velocity measurement using electrostatic sensor matrix in gas-solid two-phase pipe flow," Flow Meas. Instrum., vol. 27, pp. 104-112, Oct., 2012.

[13] Z. Kan, X. Wang, and S. Zhang, "Study on the spatial filtering and sensitivity characteristic of inserted electrostatic sensors for the measurement of gas-solid two-phase flow parameters," Flow Meas. Instrum., vol. 30, pp. 26-33, Apr. 2013.

[14] Z. Chen, X. Tang, Z. Hu, and Y. Yang, "Investigations into sensing characteristics of circular thin-plate electrostatic sensors for gas path monitoring," Chinese J. Aeronaut., vol. 27, no. 4, pp. 812-820, 2014.

[15] L. Peng, Y. Zhang, and Y. Yan, "Characterization of electrostatic sensors for flow measurement of particulate solids in square-shaped pneumatic conveying pipelines," Sensor Actuat. A-Phys, vol. 141, no. 1, pp. 59-67, 2008.

[16] Y. Yan, B. Byrne, S. Woodhead, and J. Coutlhard, "Velocity measurement of pneumatically conveyed solids using electrodynamic sensors," Meas. Sci. Technol., vol. 6, no. 5, pp. 515-537, 1995.

[17] Y. Yan, and B. Byren, "Sensing field homogeneity in mass flow rate measurement of pneumatically conveyed solids," Flow Meas. Instrum., vol. 6, no. 2, pp. 115-119, 1995.

[18] M. Abdalla, J. Zhang, R. Cheng, and D. Xu, "Non-uniformity spatial sensitivity of an electrostatic sensor," in Proc. Int. Conf. Network Security Comm. Eng., pp. 563-566, Hong Kong, China, 25-26, Dec. 2014.

[19] S. Zhang, Y. Yan, X. Qian, and Y. Hu, "Mathematical modeling and experimental evaluation of electrostatic sensor arrays for the flow measurement of fine particles in a square-shaped pipe," IEEE Sens. J., vol. 16, no. 23, pp. 8531-8541, 2016.

[20] J. Adams, M. Fairweather, and J. Yao, "Modelling and simulation of particle re-suspension in a turbulent square duct flow," Comput. Chem. Eng., vol. 35, no. 5, pp. 893-900, 2011.

[21] D. J. Griffiths, "Introduction to electrodynamics," Addison-Wesley, 4th ed., pp. 124-130, 2012.

[22] S. Matsusaka, H. Maruyama, T. Matsuyama, and M.; Ghadiri, "Triboelectric charging of powders: A review," Chem. Eng. Sci, vol. 65, no. 22, pp. 5781-5807, 2010. 